\title{
Subcutaneous Emphysema in Acute Asthma: A Cause for Concern?
}

\author{
Patrick D Mitchell MRCPI, Thomas J King MD MRCPI, and Donal B O'Shea MD FRCPI
}

\begin{abstract}
Pneumomediastinum has been described in patients with asthma. In this case report, we describe a young patient who presented to our medical assessment unit with an asthma exacerbation and progressive dyspnea. The patient developed pneumomediastinum, a rare complication of an asthma exacerbation. Pneumomediastinum is usually characterized by chest pain, dyspnea, and neck swelling caused by subcutaneous emphysema. Although the condition is usually benign and treatment is primarily supportive, surgical intervention may be needed if the patient develops hemodynamic compromise or respiratory failure through mechanisms similar to those seen in a tension pneumothorax. Key words: pneumomediastinum; asthma; dyspnea. [Respir Care 2015;60(8):e141-e143. (C) 2015 Daedalus Enterprises]
\end{abstract}

\section{Introduction}

Clinical literature reports that the peak prevalence of pneumomediastinum occurs in the second to fourth decades of life. This reflects the predisposing factors that increase the risk of developing pneumomediastinum. These factors include a higher incidence of trauma, diving, cocaine inhalation, strenuous physical exertion, and obstructive lung diseases such as asthma. Marked forced inhalation as occurs in asthma exacerbations can lead to mechanical trauma to the lung, resulting in the development of pneumomediastinum.

\section{Case Report}

A young patient with established asthma presented to our medical assessment unit complaining of increased wheezing and shortness of breath for $2 \mathrm{~d}$. The patient was a 19-y-old male student who had never been admitted

The authors are affiliated with the Department of Endocrinology and General Internal Medicine, St Columcille's Hospital, Loughlinstown, County Dublin, Ireland.

The authors have disclosed no conflicts of interest.

Correspondence: Patrick D Mitchell MRCPI, Department of Endocrinology and General Internal Medicine, St Columcille's Hospital, Loughlinstown, County Dublin, Ireland. E-mail: patrickdavidmitchell@ gmail.com.

DOI: $10.4187 /$ respcare. 03750 overnight to a hospital and who had no exacerbations for $>4 \mathrm{y}$. He was diagnosed with asthma $7 \mathrm{y}$ earlier by a pediatric respiratory specialist. The patient's only medication was a short-acting, inhaled $\beta$ agonist, which was taken periodically. He was a non-smoker with no other medical or surgical history and denied taking illicit drugs. The patient had never traveled abroad.

Admission vitals revealed a breathing frequency of 22 breaths/min, a blood pressure of 126/72 $\mathrm{mm} \mathrm{Hg}$, and a regular pulse rate of 87 beats $/ \mathrm{min}$. The patient was apyrexial, and $\mathrm{S}_{\mathrm{pO}_{2}}$ on room air was $100 \%$. Cardiovascular, gastrointestinal, and musculoskeletal examination was unremarkable. Respiratory examination was remarkable only for both an inspiratory and expiratory polyphonic wheeze. Peak flow was measured at $190 \mathrm{~L} / \mathrm{min}$. Routine blood tests were normal. His initial arterial blood gas (ABG) test was within normal range. An initial chest radiograph was normal (Fig. 1).

The patient was diagnosed as having an asthma exacerbation. We initiated standard treatment, including regular nebulized salbutamol and ipratropium, oral prednisolone, and peak flows measured every $2 \mathrm{~h}$. Over the course of the next few hours, the patient deteriorated clinically. The breathing frequency increased to 40 breaths $/ \mathrm{min}$, and the patient developed a sinus tachycardia of 110 beats/min. His blood pressure remained stable. His $\mathrm{S}_{\mathrm{pO}_{2}}$ was $90 \%$ with supplementary oxygen $\left(\mathrm{F}_{\mathrm{IO}_{2}}\right.$ of 0.40$)$.

On re-examination, the patient was found to have developed bilateral supraclavicular subcutaneous emphysema. The ABG analysis was repeated and revealed a $\mathrm{pH}$ 


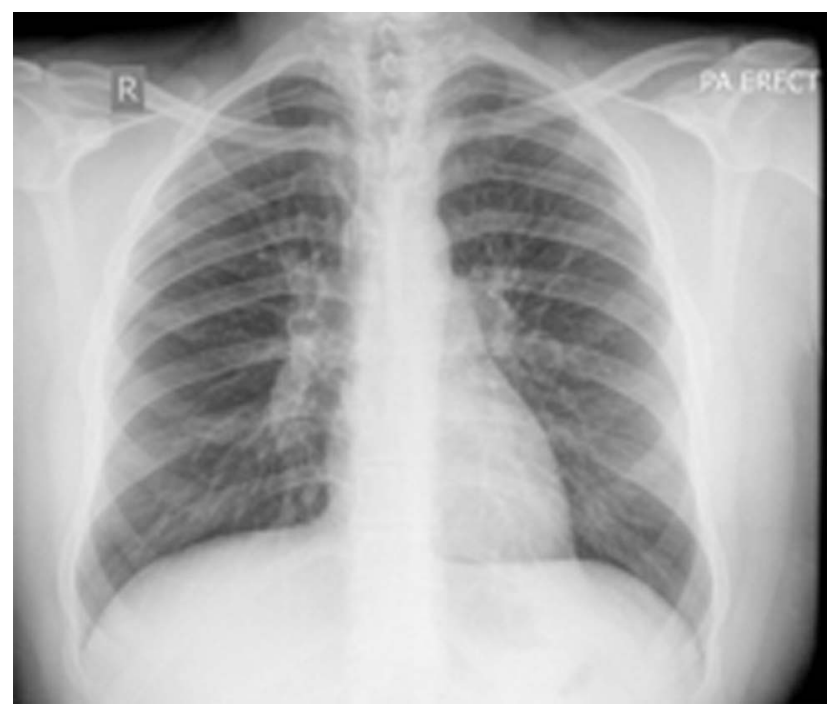

Fig. 1. Initial presenting chest radiograph. This was reported as normal.

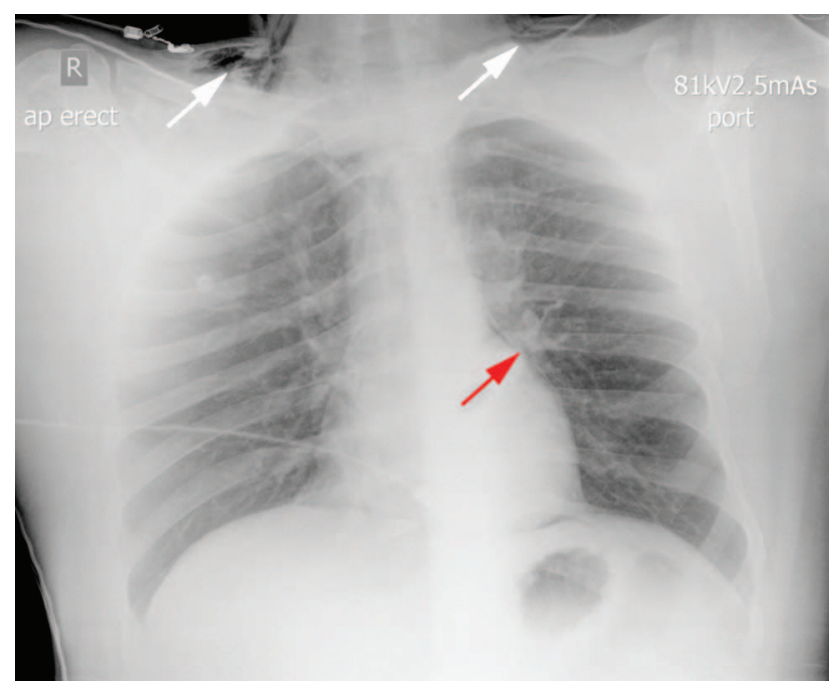

Fig. 2. Chest radiograph $12 \mathrm{~h}$ later. The arrows highlight the new finding of pneumomediastinum (red arrow) and supraclavicular subcutaneous emphysema (white arrows).

of 7.33, a $\mathrm{P}_{\mathrm{aO}_{2}}$ of $61 \mathrm{~mm} \mathrm{Hg}, \mathrm{a}_{\mathrm{aCO}_{2}}$ of $59 \mathrm{~mm} \mathrm{Hg}$, sodium bicarbonate at $23.6 \mathrm{mmol} / \mathrm{L}$, and a base excess of +4 mmol/L. A portable chest radiograph (Fig. 2) was done and was remarkable for bilateral supraclavicular subcutaneous emphysema and pneumomediastinum. This had developed in the time since the initial chest radiograph (see Fig. 1). A decision was then made to escalate management, so the patient was transferred to the ICU, where intubation using rapid sequence induction was performed, and he was placed on positive pressure ventilation. The decision to perform an intubation as opposed to noninvasive ventilation was based on: (1) increasing drowsiness and exhaustion, (2) the patient's ABG results, and (3) the presence of pneumomediastinum. We were concerned that positive airway pressure would precipitate the development of tension pneumomediastinum, so intubating the patient would allow better control of his airways and easier chest tube placement in an emergency situation. The patient was closely monitored for any hemodynamic compromise that could be caused by the positive airway pressures in the setting of pneumomediastinum. The patient was kept intubated for $24 \mathrm{~h}$ before being extubated and placed on the ward. He was discharged home $3 \mathrm{~d}$ later.

\section{Discussion}

Blunt trauma, iatrogenic injury, esophageal perforation (Boerhaave syndrome), the Valsalva maneuver (eg, child birth), strenuous exercise, shouting, barotrauma (diving, intubation), cocaine inhalation, asthma exacerbations, COPD, and interstitial lung disease have all been described as causing pneumomediastinum. ${ }^{1,2}$ Pneumomediastinum rarely leads to clinically important complications except when caused by trauma or Boerhaave syndrome. More commonly, the associated or precipitating condition underlying pneumomediastinum may be the cause of significant illness, as in this case (asthma). The generally accepted explanation for the development of pneumomediastinum is that free air tracks from ruptured alveoli along peribronchial vascular sheaths toward the hilum of the lung. From there, it extends proximally within the mediastinum; this is explained by the Macklin effect. The Macklin effect, first described in 1939, highlights the sequence of events in the development of pneumomediastinum as follows: alveolar rupture, which allows air to track along the bronchovascular fascia, where it accumulates and causes pneumomediastinum. ${ }^{3}$ The dissection of free air may not be confined solely to the mediastinum. Anatomically, the mediastinum communicates with the submandibular space, the retropharyngeal space, and vascular sheaths within the neck. Two routes of communication within the retroperitoneum have been described. One route is via a tissue plane extending through the sternocostal attachment to the diaphragm; another route is through the periaortic and periesophageal fascial planes. Air present within the mediastinum may dissect through these tissue planes, causing pneumopericardium, pneumothorax, subcutaneous emphysema, pneumoperitoneum, or pneumoretroperitoneum.

In severe asthma, pneumomediastinum develops because of overexpansion of the distal airways due to the obstruction in the minor airways with subsequent alveolar rupture. Because of the pressure difference, the air in the pulmonary interstitium moves in the centripetal direction from the pulmonary parenchyma toward the mediastinum as described above. ${ }^{4}$ Although pneumomediastinum is generally a benign and self-limiting condition that responds to conservative therapy, its concurrence with pneumothorax 
may prove fatal during a serious asthma attack. Some serious complications of pneumomediastinum include high blood pressure and/or bilateral pneumothorax, as well as cardiac compression and high pressure, causing a reduction in cardiac output and hemodynamic compromise. There is a $10 \%$ probability of pneumomediastinum evolving into a pneumothorax, whereas the converse never happens. As misdiagnosis and delayed treatment of pneumomediastinum can lead to a tension pneumothorax and ultimately to death, it is imperative that patients are monitored for at least $48 \mathrm{~h}$. Tension pneumomediastinum has rarely been reported, in which elevated mediastinal pressure leads to diminished cardiac output because of direct cardiac compression or reduced venous return, which can require surgical decompression. ${ }^{5}$ When extensive subcutaneous and mediastinal gas is present, airway compression may also occur, which may necessitate surgical or radiological intervention.

This case demonstrates that a high level of clinical suspicion and pathophysiological knowledge of the compli- cations of pneumomediastinum is required when treating patients with asthma exacerbations.

\section{REFERENCES}

1. Dyer J, Jones S. Vomiting-induced surgical emphysema and pneumomediastinum: a self-remitting or life-threatening condition? BMJ Case Rep 2011;2011:bcr1220103608.

2. Ojima H, Fukai Y, Soda M, Kuwano H. Asymptomatic spontaneous cervical and mediastinal emphysema. BMJ Case Rep 2012;2012: bcr2012006248.

3. Wintermark M, Schnyder P. The Macklin effect: a frequent etiology for pneumomediastinum in severe blunt chest trauma. Chest 2001; 120(2):543-547.

4. Maunder RJ, Pierson DJ, Hudson LD. Subcutaneous and mediastinal emphysema. Pathophysiology, diagnosis, and management. Arch Intern Med 1984;144(7):1447-1453.

5. Dajer-Fadel WL, Argüero-Sánchez R, Ibarra-Pérez C, Navarro-Reynoso FP. Systematic review of spontaneous pneumomediastinum: a survey of 22 years' data. Asian Cardiovasc Thorac Ann 2014;22(8): 997-1002. 